\title{
A importância da reabilitação oral estética na alteração de forma e cor dos dentes: relato de caso clínico
}

The importance of oral aesthetic rehabilitation in changing shape and color of teeth: clinical case report La importancia de la rehabilitación oral estética en la alteración de forma y color de los dientes: reporte de caso clínico

Daiany Catão FURTADO ${ }^{1}$

Eloiza Leonardo de MELO

Mayara Aragão de Lira GOMES ${ }^{3}$

Kaíse Tavares PONTES ${ }^{4}$

José Lacerda das NEVES ${ }^{5}$

Carlos Alberto de Souza CANTO

Claudio Paulo Pereira de ASSIS

Rodivan BRAZ ${ }^{8}$

\begin{abstract}
${ }^{1}$ Mestre em Ciência e Tecnologia em Saúde, Universidade Estadual da Paraíba-UEPB, 58429-500, Campina Grande-PB, Brasil
${ }^{2}$ Mestranda em Odontologia, Área de concentração em Dentística/Endodontia, Universidade de Pernambuco-UPE, 54753-220 Camaragibe-PE, Brasil

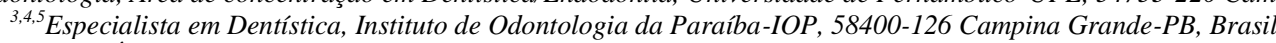

${ }^{6}$ Mestre em Odontologia, Área de concentração em Dentística/Endodontia, Universidade de Pernambuco-UPE, 54753-220 Camaragibe-PE, Brasil

7 Doutorando em Odontologia, Área de concentração em Dentística/Endodontia, Universidade de Pernambuco-UPE, 54753-220 Camaragibe-PE, Brasil

${ }^{8}$ Professor de Dentística da Universidade de Pernambuco, 54753-220, Camaragibe-PE, Brasil
\end{abstract}

\section{Resumo}

Com o aumento da exigência estética dos pacientes e o desenvolvimento de materiais e técnicas odontológicas restauradoras problemas como alteração de cor e forma tem sido melhor resolvidos garantindo naturalidade e longevidade aos procedimentos restauradores. Este trabalho objetivou apresentar um relato de caso clínico de reabilitação estética do sorriso, descrevendo protocolo clínico de laminados cerâmicos e coroas totais "metal free" desde o planejamento até a cimentação definitiva. Paciente do gênero feminino, apresentou insatisfação com a estética de seu sorriso, principalmente em relação a coloração de seus dentes. Durante avaliação clínica, constatou-se comprometimento estético dos elementos dentais 11, 12 e 21 com alteração de cor e desequilíbrio dimensional. Esses dentes tinham grande restaurações resina composta, o que os tornava opacos e sem brilho. O tratamento proposto consistiu na realização de clareamento dental e posteriormente laminados cerâmicos nos dentes 12,13, 14, 22, 23 e 24 e coroas totais "metal-free" nos elementos 11 e 21 , utilizando um sistema cerâmico a base de dissilicato de lítio. Após o relato deste caso clínico, pode-se concluir que com o advento das cerâmicas ácido sensíveis e da cimentação adesiva os tratamentos reabilitadores estéticos têm proporcionado um resultado estético e funcional satisfatório.

Descritores: Facetas Dentárias; Odontologia; Dentística Operatória.

\section{Abstract}

With increasing aesthetic requirement of patients and the development of materials and restorative dental techniques problems such as alteration of color and shape have been better solved guaranteeing naturalness and longevity to restorative procedures. This paper aimed to present a report of a clinical case of smile aesthetic rehabilitation, describing clinical protocol of ceramic laminates and total crowns "metal free" from planning to final cementation. A female patient, she was dissatisfied with the aesthetics of her smile, mainly in relation to the color of her teeth. During clinical evaluation, it was found aesthetic compromise of dental elements 11, 12 and 21 with color change and dimensional imbalance. These teeth had large composite resin restorations, which made them opaque and lackluster. The proposed treatment consisted of dental bleaching and then ceramic laminates on teeth 12,13,14,22, 23 and 24 and total metal-free crowns on elements 11 and 21 using a ceramic system based on lithium disilicate. After the report of this clinical case, it can be concluded that with the advent of the sensitive acid ceramics and adhesive cementation the aesthetic rehabilitation treatments have provided a satisfactory aesthetic and functional result.

Descriptors: Dental Veneers; Dentistry; Dentistry, Operative.

\section{Resumen}

Con el aumento de la exigencia estética de los pacientes y el desarrollo de materiales y técnicas odontológicas restauradoras problemas como alteración de color y forma han sido mejor resueltos garantizando naturalidad y longevidad a los procedimientos restauradores. Este trabajo objetivó presentar un relato de caso clínico de rehabilitación estética de la sonrisa, describiendo protocolo clínico de laminados cerámicos y coronas totales "metal free" desde la planificación hasta la cementación definitiva. Paciente del género femenino, presentó insatisfacción con la estética de su sonrisa, principalmente en relación a la coloración de sus dientes. Durante la evaluación clínica, se constató un compromiso estético de los elementos dentales 11, 12 y 21 con alteración de color y desequilibrio dimensional. Estos dientes tenían grandes restauraciones resina compuesta, lo que los hacía opacos y sin brillo. El tratamiento propuesto consistió en la realización de blanqueamiento dental y posteriormente laminados cerámicos en los dientes 12, 13, 14, 22, 23 y 24 y coronas totales "metal-free" en los elementos 11 y 21 , utilizando un sistema cerámico a base de disilicato de litio. Después del relato de este caso clínico, se puede concluir que con el advenimiento de las cerámicas ácido sensibles y de la cementación adhesiva los tratamientos rehabilitadores estéticos han proporcionado un resultado estético y funcional satisfactorio.

Descriptores: Coronas con Frente Estético; Odontología; Operatoria Dental.

\section{INTRODUÇÃO}

Problemas como alteração de forma e cor têm se tornado atualmente as principais queixas no cotidiano clínico. Portando com o crescimento da exigência estética e $\mathrm{o}$ acesso a tratamentos reabilitadores minimamente invasivos essas condições podem ser resolvidas com menos danos à estrutura dentária, resultando em reabilitações duradouras, funcionais e com aspecto natural ${ }^{1}$.
Diante disto, o desenvolvimento de materiais e técnicas odontológicas restauradoras tem sido incentivado expressivamente pela constate busca pela naturalidade dos resultados dos procedimentos restauradores $^{2}$. As cerâmicas, especialmente aquelas ácido sensíveis, são atualmente materiais de eleição para estes tratamentos de alta performance estética devido às suas excelentes propriedades, como 
biocompatibilidade, longevidade, estabilidade de cor e propriedades mecânicas que biomimetizam o esmalte dentário ${ }^{3}$. Entretanto, com todos estes benefícios das cerâmicas, para garantir longevidade ao trabalho o cirurgião-dentista deve avaliar criteriosamente alguns pontos como quantidade de desgate do preparo dental, possíveis falhas no término da moldagem e escolha da cerâmica de acordo com o substrato a ser trabalhado e o protocolo de cimentação ${ }^{1}$.

Por outro lado, situações clínicas em que há considerável perda da estrutura dental, a indicação de coroas com sistemas cerâmicos totalmente puros ("metal free") mostra-se como excelente alternativa restauradora. Elas apresentam estética superior às coroas metalocerâmicas, devido à possibilidade da realização de estratificação de cores de modo mais eficiente, possibilitando um aspecto mais natural e harmonioso com os dentes adjacentes, entretanto o protocolo de cimentação nestes casos difere do protocolo para cerâmicas ácido sensíveis ${ }^{1}$.

Os laminados cerâmicos, são cerâmicas ácido sensíveis que possibilitam reabilitações estéticas com o mínimo desgaste da estrutura dentária. Através de estudos clínicos e laboratoriais verificou-se que os laminados cerâmicos são restaurações duráveis e resistentes as situações clínicas quando devidamente indicados. Além do sucesso clínico, é um procedimento que proporciona alta resolução estética ${ }^{4}$.

Diante da problemática atual e da exigência estética dos pacientes é importante a discussão de casos que contribuam para a atualização do Cirurgião-Dentista. Assim, o objetivo deste trabalho foi apresentar um relato de caso clínico de reabilitação estética do sorriso, descrevendo protocolo clínico de laminados cerâmicos e coroas totais "metal free" desde o planejamento até a cimentação definitiva.

\section{CASO CLÍNICO}

Paciente do gênero feminino relatou insatisfação com a estética de seu sorriso, especialmente em relação a coloração de seus dentes. Através avaliação clínica, constatou-se comprometimento estético dos elementos dentais 11 , 12 e 21 com alteração de cor e comprometimento do movimento protrusivo. Observamos também restaurações extensas em resina composta opaca e sem brilho (Figuras 1 e 2).

O tratamento proposto para a paciente consistiu no emprego de clareamento dental em consultório para preparo do substrato dentário em con e posteriormente de laminados cerâmicos nos dentes $12,13,14,22,23$ e 24 e coroas totais metal free nos elementos 11 e 21, utilizando cerâmica ácida sensível à base de dissilicato de lítio. $\mathrm{O}$ clareamento dental a base de peróxido de hidrogênio a 35\% (Total Blanc
Office, Nova DFL, Rio de Janeiro, RJ, Brasil) foi dividido em duas sessões com um intervalo de um mês entre uma e outra (Figura 3). Após o clareamento, realizou-se moldagem dos arcos superior e inferior utilizando silicone de adição (Futura AD, Nova DFL, Rio de Janeiro, RJ, Brasil), para realização de enceramento diagnóstico.

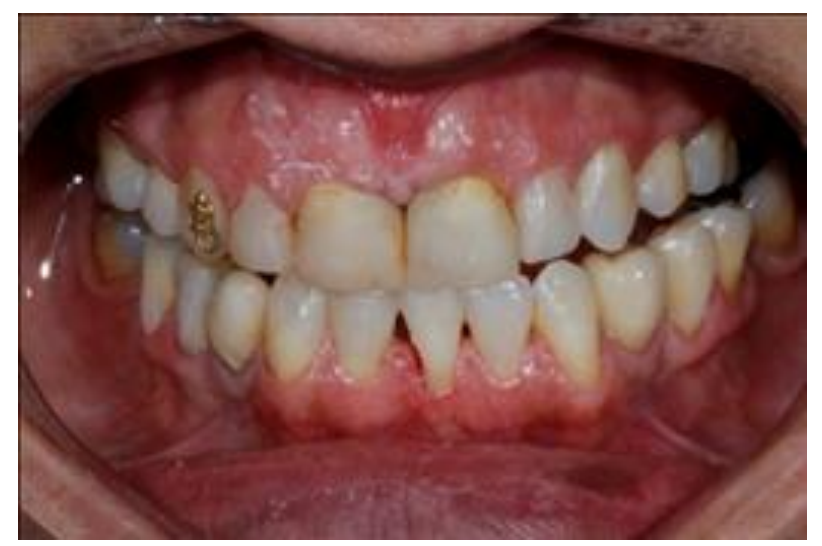

Figura 1: Movimento protrusivo evidenciando interferencia oclusal no dente 43.

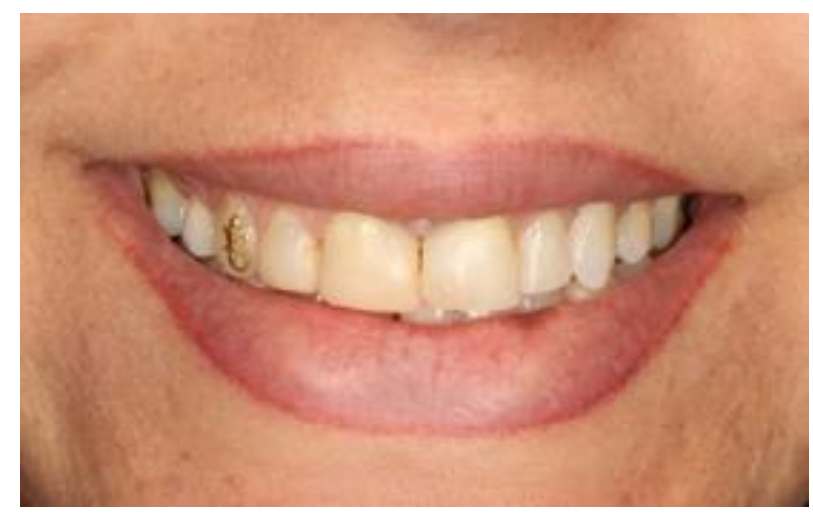

Figura 2: Restaurações extensas e opacas em resina composta e dentes incisivos centrais com comprimento inadequado.

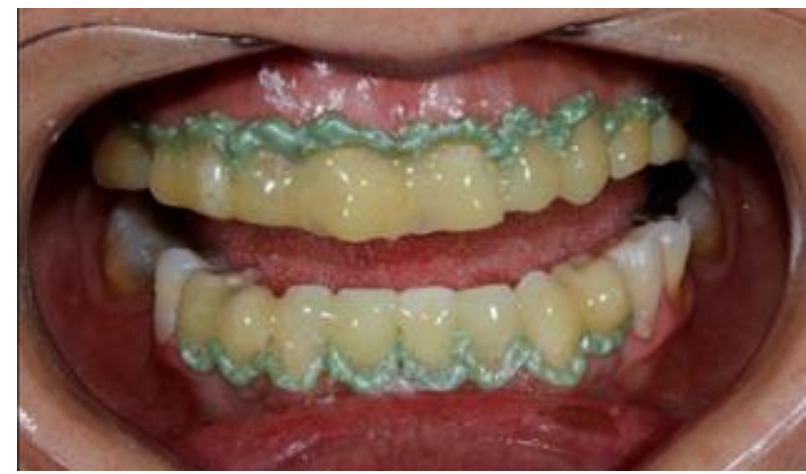

Figura 3: Clareamento Dental.

O mock-up foi realizado com objetivo de avaliação estética e funcional, onde foi utilizada resina bisacrílica (Structur 2 SC, Voco, Porto Alegre, RS, Brasil) na cor A2 (Figuras 4 e 5). Os excessos foram removidos e os ajustes oclusal e estético realizados. Durante essa etapa além da avaliação do profissional, a paciente teve oportunidade de avaliar o planejamento da reabilitação estética. 


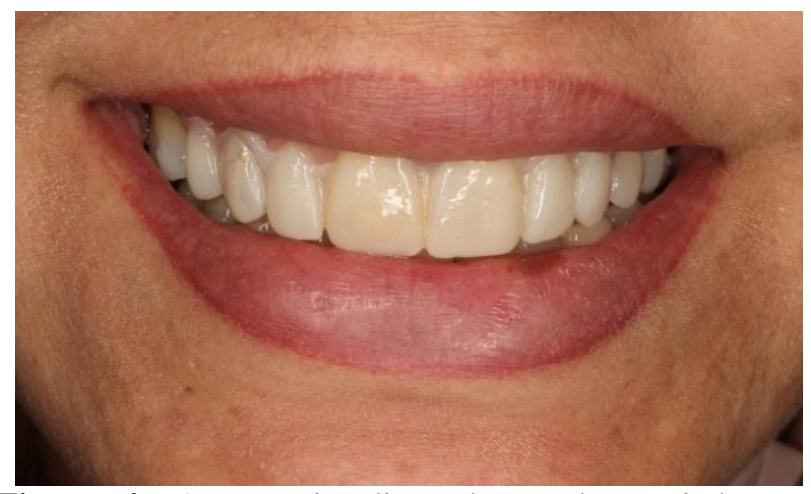

Figura 4: Aspecto imediato do mock-up ainda sem acabamento, porém com o comprimento dos dentes corrigidos melhorando a harmonia do sorriso.

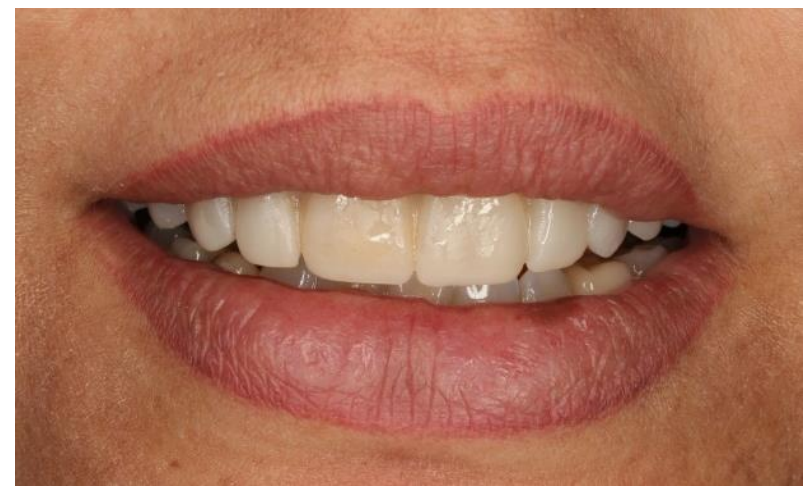

Figura 5: Lábios em repouso evidenciando a importância do aumento da borda incisal para a melhoria da harmonia do sorriso.

Os preparos foram realizados com pontas diamantadas de granulações finas e ultrafinas para a remoção dos ângulos vivos e áreas retentivas, mantidos em nível de esmalte, melhorando assim, o eixo de inserção e adaptação para os laminados cerâmicos (Figura 6).

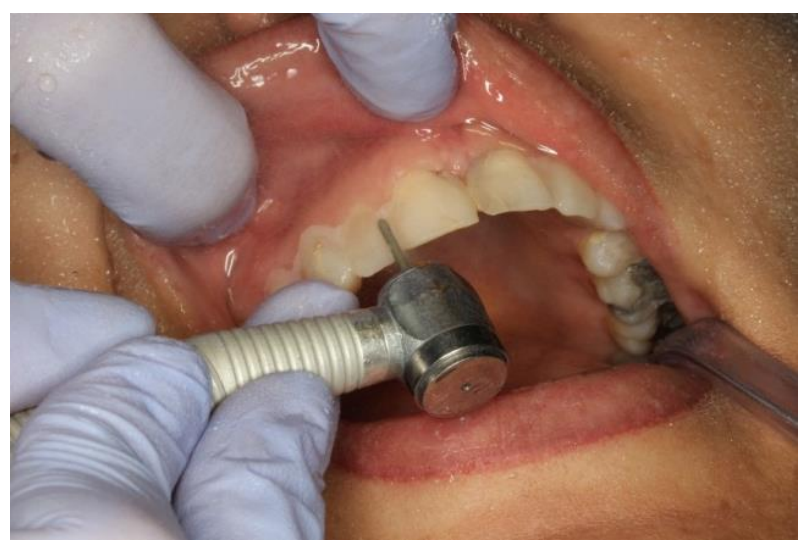

Figura 6: Preparo dos laminados sobre o mock-up.

Devido à presença de grandes restaurações de resina composta nos elementos 11 e 21, planejou-se para os mesmos preparo para coroa total. Para isto foi realizada uma canaleta de orientação na região cervical das faces vestibular e palatina posicionada a 2 milímetros supragengival com ponta diamantada esférica $n^{\circ} 1014$ (KG Sorensen, São Paulo, SP, Brasil) sob inclinação de aproximadamente $45^{\circ} \mathrm{em}$ relação ao longo eixo do dente e profundidade média de 0,7 milímetros. Na sequência, foram realizados três sulcos de orientação na face vestibular do dente, no sentido cervico-incisal, levando-se em consideração a inclinação da superfície vestibular. Assim o desgaste foi realizado respeitando os três planos dentais (cervical, médio e incisal) com uma ponta diamantada n 3216 (KG Sorensen, São Paulo, SP, Brasil) e em seguida os sulcos de orientação foram unidos com a mesma ponta diamantada. A concavidade palatina foi confeccionada com ponta diamantada $n^{\circ} 3118$ (KG Sorensen, São Paulo, SP, Brasil). Os pontos de contatos foram rompidos com ponta diamantada afilada $\mathrm{n}^{\circ} 2200$ (KG Sorensen, São Paulo, Brazil) e as áreas interproximais foram preparadas com a ponta diamantada $\mathrm{n}^{\circ}$ 3216. A redução incisal também foi realizada com a ponta diamantada $\mathrm{n}^{\circ} 3216$ posicionada em angulação de $45^{\circ}$ para lingual com profundidade de 2 milímetros. Para o preparo subgengival, foi inserido fio retrator \#00 (Ultrapack, Ultradent, Estados Unidos) e utilizou-se a ponta diamantada $\mathrm{n}^{\circ} 3216$, dando ao término em forma de ombro arredondado uma profundidade de 0,5 milímetros intrassucular. Por fim, os preparos foram finalizados (Figura 7) com acabamento utilizando ponta diamantada $\mathrm{n}^{\circ}$ 4138FF (KG Sorensen, São Paulo, Brasil).

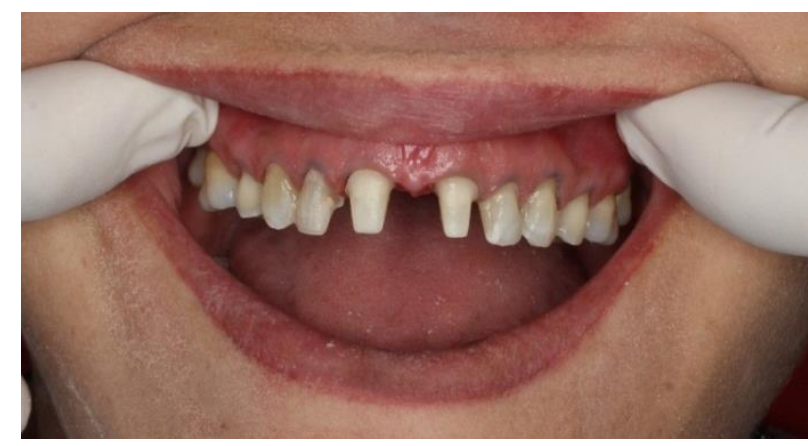

Figura 7: Preparos Finalizados

A moldagem foi realizada com silicone de adição (Futura AD, Nova DFL, Rio de Janeiro, RJ, Brasil) pela técnica de dupla moldagem. Para registrar o término com precisão, realizou-se o afastamento gengival com fio retrator \#00 (Ultrapack, Ultradent, Estados Unidos) inserido no interior do sulco com espátula de inserção de fio retrator.

Após a seleção da moldeira foi realizada a primeira moldagem com silicone de adição pasta densa. A presa do material foi aguardada para a remoção da moldeira. O fio retrator foi removido, levou-se o silicone de adição fluido aos preparos e à moldeira, inserindo-a em posição na boca. Posteriormente a moldeira foi removida da boca e o molde foi lavado em água corrente e seco para a verificação da correta reprodução das estruturas.

$\mathrm{Na}$ sequência procedeu-se com a seleção da cor empregando escala de cores VITA (Vitapan, Vita 
Zahnfabrik, Bad Säckingen, Alemanha), a cor selecionada foi A1. Os provisórios dos elementos 11 e 21 foram confeccionados com dente de estoque e resina acrílica Duralay cor 66 (Reliance Dental Manufacturing, Estados Unidos) e para os elementos $12,13,14,22,23$ e 24 foram confeccionados provisórios de resina bisacrílica (Structur $2 \mathrm{SC}$, Voco, Porto Alegre, RS, Brasil), utilizando o molde de silicone de adição confeccionado anteriormente para a realização do mock-up.

O protocolo fotográfico também foi enviado ao laboratório de prótese juntamente com o molde para melhor comunicação com o ceramista, permitindo a ele conhecer as características e detalhes do sorriso da paciente.

$\mathrm{Na}$ sessão clínica seguinte após a remoção dos provisórios e profilaxia dos preparos, foi realizada a prova das coroas e dos laminados cerâmicos, para análise da cor, forma, adaptação marginal e para aprovação da paciente.

O protocolo de cimentação adesiva iniciou-se pela prova do cimento com as pastas de prova "Tryin", que foram dispensadas na face interna dos laminados cerâmicos e estes posicionados sobre os dentes preparados. Sendo portanto escolhida a cor A1. As superfícies internas dos laminados foram condicionadas com ácido fluorídrico $10 \%$ durante 20 segundos. Após o tempo de tratamento recomendado, as superfícies foram lavadas por 60 segundos e secas, em seguida os laminados foram condicionados com ácido fosfórico $37 \%$ por 60 segundos. As peças receberam novamente abundante jato de ar/água para completa remoção de resíduos durante 60 segundos. Logo após, as peças foram secas, aplicou-se uma camada de silano (Silano, Angelus, Londrina, PR, Brasil) aguardando a evaporação por 1 minuto e uma camada de adesivo Single Bond Universal (3M, Sumaré, SP, Brasil) seguida de jato de ar. A cimentação dos laminados com as coroas em posição, utilizando cimento resinoso fotopolimerizável Allcem Veneer (FGM, Joinville, SC, Brasil).

As coroas dos elementos 11 e 21 foram cimentadas com cimento resinoso Enforce Dual (Dentsply, Rio de Janeiro, RJ, Brasil). As coroas foram condicionadas internamente com ácido fluorídrico a $10 \%$ por 20 segundos, seguindo-se com lavagem abundante com água e posterior secagem com ar. Logo depois, aplicou-se o agente de silanização por 1 minuto e realizou-se a hibridização das superfícies dentárias. Carregadas com o cimento, as coroas foram levadas aos dentes e inseridas aos preparos, removeram-se os excessos de material para posterior fotopolimerização.

$\mathrm{O}$ ajuste final da oclusão foi executado em Máxima Interscupidação Habitual de forma criteriosa, sendo também verificados os movimentos de protrusão e lateralidade. O aspecto final está evidenciado na Figura 8, onde se pode observar um resultado esteticamente satisfatório.

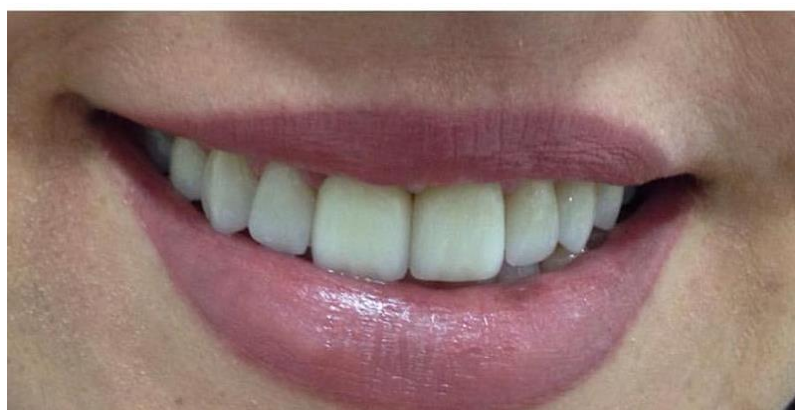

Figura 8: Aspecto final das facetas cimentadas.

DISCUSSÃO

O sucesso de uma reabilitação oral cerâmica está condicionado ao respeito a um protocolo criterioso durante a realização dos preparos dentais pelo profissional. $\mathrm{O}$ fracasso na reabilitação pode ser ocasionado pela inabilidade do operador ou até por negligência do mesmo com a realização de uma técnica adequada. O preparo dental satisfatório deve apresentar uma quantidade de desgaste correta, condições mecânicas de estabilidade e retenção para a adaptação da coroa protética e longevidade. Portanto, apesar da evolução dos cimentos, a forma geométrica dos preparos tem papel fundamental no sucesso clínico de reabilitações cerâmicas ${ }^{5}$.

A profundidade dos preparos é determinada pela alteração de cor dos elementos dentários envolvidos, extensão das antigas restaurações de resina composta e posição dos dentes no arco dental ${ }^{6}$. No caso clínico apresentado, indicou-se coroa total "metal free" nos incisivos centrais devido à presença de extensas restaurações de resina composta insatisfatórias e laminados cerâmicos nos incisivos laterais, caninos e primeiros pré-molares, devido à necessidade de pequenas correções de cor e forma.

Os laminados cerâmicos têm diversas indicações, destacando-se a substituição de restaurações deficientes e a transformação de elementos dentários. Este tratamento restaurador apresenta inúmeras vantagens, tais como: preparo conservador quando comparado ao prepara para coroa total, excelente estética, alta resistência ao desgaste e alta compatibilidade ao periodonto devido ao pouco acúmulo de placa e a facilidade de higienização ${ }^{7}$. Este procedimento tornou-se muito popular nos últimos anos devido a seu índice de sucesso, o qual pode chegar a até $95 \%$ num período de 5 anos, e as suas características conservadoras .

Através do enceramento diagnóstico, tem-se a previsibilidade do tratamento por meio da confecção do mock-up. O mock-up é realizado nos dentes que receberão as restaurações cerâmicas de forma provisória seguindo o mesmo padrão estético das restaurações cerâmicas que serão cimentadas, ele pode ser confeccionado de forma direta com resina composta ou de forma indireta utilizando resina bis- 
acrílica $^{9}$. No caso clínico apresentado o mock-up foi confeccionado de maneira indireta com resina bisacrílica.

Um relevante fator ligado ao sucesso clínico é o pré-tratamento da superfície interna da peça protética previamente à cimentação. $\mathrm{O}$ pré-tratamento varia de acordo com o material da prótese, podendo ser classificado em mecânico ou químico. A finalidade desta etapa é elevar a rugosidade superficial e consequentemente aumentar a adesão por retenção micromecânica. Nas cerâmicas como o IPS e.max ${ }^{\circledR}$ a força de adesão é satisfatória quando a peça é tratada combinando-se ácido hidrofluorídrico e silano ${ }^{10}$. Na reabilitação apresentada, foi realizado pré-tratamento químico das peças protéticas e do substrato dentário.

A cimentação das próteses cerâmicas também corresponde a uma importante etapa para o sucesso da reabilitação. Existem diversos agentes cimentantes e possibilidades de tratamento da superfície, portanto a seleção do cimento deve ser embasada em conhecimento científico ${ }^{11}$.

Nos casos de laminados cerâmicos são utilizadas pastas de prova dos cimentos com diferentes colorações previamente a cimentação. Esta etapa é realizada para que não haja equívoco na seleção de cor do cimento e consequentemente prejuízo no resultado estético final ${ }^{12}$.

No caso clínico apresentado, utilizou-se para cimentação dos laminados cerâmicos cimento resinoso fotopolimerizável, o qual é considerado o mais indicado para nesses casos. Os cimentos quimicamente ativados ou duais apresentam na sua composição a amina terciária como ativador químico, tal substância pode apresentar alteração de cor com o decorrer do tempo, provocando um manchamento marginal da interface dente-restauração, contraindicando estes cimentos para os laminados cerâmicos ${ }^{6}$.

Já na cimentação das coroas metal free, utilizou-se cimento resinoso dual. A seleção desse cimento deve-se a possibilidade de ativação dupla, na qual existe associação dos processos de ativação química e de fotoativação, melhorando significativamente as propriedades físicas e mecânicas. O cimento dual apresenta melhor desempenho de retenção de peças protéticas quando comparado aos cimentos de ativação única $\mathrm{e}$ tradicional ${ }^{13}$.

\section{CONCLUSÃO}

As reabilitações orais realizadas com restaurações cerâmicas têm demonstrado excelente resultado estético e longevidade, desde que respeitado um protocolo clínico rigoroso baseado em princípios de preparo, moldagem e cimentação. Após o relato deste caso clínico, pode-se concluir que é possível restabelecer a estética e função em indivíduos insatisfeitos com seu sorriso, através da realização de restaurações em cerâmica.

\section{REFERÊNCIAS}

1. Lima AF, Carvalho JFO, Cravo FL. Restaurações cerâmicas em dentes anteriores: simples realização? Rev Dental Press Estét, 2010; 7(4):88-96.

2. Carvalheira TB, Goyata FR, Rodrigues CRT, Souza MCA. Resolução estética em dentes anteriores com coroas totais livres de metal-relato de caso clínico. Int j dent. 2010;9(2):102-6.

3. Aquino APT, Cardoso PC, Rodrigues MB, Takano AE, Porfírio W. Facetas de porcelana: solução estética e funcional. Clin int $\mathrm{j}$ braz dent. 2009;5(2):142-52.

4. Peumans, M.; Van Meerbeek, B.; Lambrechts, P.; Vanherle, G. Porcelain veneers: a review of the literature. J Dent. 2000;28(3):163-77.

5. Busato ALS, Hernandez PAG, Macedo RP. Dentística: restaurações estéticas. 2. ed. São Paulo: Artes Médicas; 2002.

6. Conceição EN. Dentística: saúde e estética. 2. ed. Porto Alegre: Artmed; 2007.

7. Farias FAR, Feltrin PP, Zanetti AL, Inoue RT. Preparo dentário para coroa metalocerâmica em dentes anteriores, por meio da técnica de referência Inoue \& Zanetti. RGO Rev Gaúcha Odontol. 2011;59(Suppl 0):81-8.

8. Massing NG, Bellatoto LB, Magagnin C, Silva SBA, Busato ALS, Barbosa NA. Facetas estéticas em porcelana. Rev Ibero-Americana Odontol Estet Dent. 2006;5(18):136-41.

9. Calixto LR, Bandeca MC, Andrade MF. Enceramento diagnóstico: previsibilidade no tratamento estético indireto. Rev Dental Press Estét. 2011;8(3):26-37.

10.Stewart GP, Jain P, Hodges J. Shear bond strength of resin cements to both ceramic and dentin. J Prosthet Dent. 2002;88(3):277-84.

11.Valle AL, Martin LM, Chidiak-Tawil R, Pimentel GHD, Rodrigues MGS, Ramos MB et al. Sistemas cerâmicos atuais: revisão de literatura. Rev Dental Press Estét. 2010;7(1):106-7.

12.Rosalem C, Hirata R, Andrade OS, Borges G, Celestrino M. Pastas de prova em laminados cerâmicos: aplicação clínica para alcançar o resultado estético. Clin int $\mathrm{j}$ braz dent. 2010;6(2):210-21.

13. Guedes LLS, Matto ECG, Zani SM, Prates LHM, Chain MC. Avaliação das propriedades mecânicas de cimentos resinosos convencionais e autocondicionantes. Rev Odontol Unesp.2008; 37(1):85-9. 


\section{CONFLITO DE INTERESSES}

Os autores declaram não haver conflitos de interesse.

\section{AUTOR PARA CORRESPONDENCIA}

Eloiza Leonardo de Melo

eloizaleonardo@gmail.com

Submetido em 25/06/2018

Aceito em 04/10/2018 